\title{
Rademacher functions in Cesàro type spaces
}

by

\author{
Sergei V. Astashkin (Samara) and Lech Maligranda (Luleå)
}

\begin{abstract}
The Rademacher sums are investigated in the Cesàro spaces $\operatorname{Ces}_{p}(1 \leq$ $p \leq \infty)$ and in the weighted Korenblyum-Kreln-Levin spaces $K_{p, w}$ on [0,1]. They span $l_{2}$ space in $\operatorname{Ces}_{p}$ for any $1 \leq p<\infty$ and in $K_{p, w}$ if and only if the weight $w$ is larger than $t \log _{2}^{p / 2}(2 / t)$ on $(0,1)$. Moreover, the span of the Rademachers is not complemented in $\operatorname{Ces}_{p}$ for any $1 \leq p<\infty$ or in $K_{1, w}$ for any quasi-concave weight $w$. In the case when $p>1$ and when $w$ is such that the span of the Rademacher functions is isomorphic to $l_{2}$, this span is a complemented subspace in $K_{p, w}$.
\end{abstract}

1. Introduction and preliminaries. Consider the Rademacher functions on $[0,1]$ defined by $r_{k}(t)=\operatorname{sign}\left(\sin 2^{k} \pi t\right), k \in \mathbb{N}, t \in[0,1]$, and the set of Rademacher sums

$$
R_{n}(t)=\sum_{k=1}^{n} a_{k} r_{k}(t), \quad a_{k} \in \mathbb{R} \quad \text { for } k=1, \ldots, n, \text { and } n \in \mathbb{N} .
$$

The behaviour of Rademacher sums in $L_{p}=L_{p}[0,1]$ spaces is well known (cf. [6. p. 10]). In particular, it follows from the classical Khintchine inequality that there exist constants $A_{p}, B_{p}>0$ such that for any sequence $\left\{a_{k}\right\}_{k=1}^{n}$ of real numbers and any $n \in \mathbb{N}$ we have

$$
A_{p}\left(\sum_{k=1}^{n}\left|a_{k}\right|^{2}\right)^{1 / 2} \leq\left\|R_{n}\right\|_{L_{p}[0,1]} \leq B_{p}\left(\sum_{k=1}^{n}\left|a_{k}\right|^{2}\right)^{1 / 2}, \quad 0<p<\infty,
$$

and hence the Rademacher functions $\left\{r_{n}\right\}$ span an isomorphic copy of $l_{2}$ in $L_{p}$ for every $0<p<\infty$. Moreover, the subspace $\left[r_{n}\right] \simeq l_{2}$ is complemented in $L_{p}$ for $1<p<\infty$ and is not complemented in $L_{1}$ since no complemented infinite-dimensional subspace of $L_{1}$ can be reflexive. In $L_{\infty}$ we have $\left\|R_{n}\right\|_{L_{\infty}[0,1]}=\sum_{k=1}^{n}\left|a_{k}\right|$ and so the Rademacher functions span an isometric copy of $l_{1}$, which is known to be uncomplemented in $L_{\infty}$.

2010 Mathematics Subject Classification: 46E30, 46B20, 46B42.

Key words and phrases: Rademacher functions, Cesàro function spaces, KorenblyumKrel̆n-Levin spaces, rearrangement invariant spaces, subspaces, complemented subspaces. 
In the case of rearrangement invariant spaces $X$ on $[0,1]$, Rodin and Semenov [16] proved that $\left\|\sum_{k=1}^{\infty} a_{k} r_{k}\right\|_{X} \approx\left(\sum_{k=1}^{\infty}\left|a_{k}\right|^{2}\right)^{1 / 2}$ if and only if $G \subset X$, where $G$ is the closure of $L_{\infty}[0,1]$ in the Orlicz space $L_{M}[0,1]$ generated by the function $M(u)=e^{u^{2}}-1$. Note that this Orlicz space coincides with the Marcinkiewicz space $M(\varphi)$ generated by the function $\varphi(t)=t \log _{2}^{1 / 2}(2 / t), 0<t \leq 1$, where the norm is given by $\|f\|_{M(\varphi)}=$ $\sup _{0<x \leq 1}(1 / \varphi(x)) \int_{0}^{x} f^{*}(t) d t$. Here, $f^{*}$ is the non-increasing rearrangement of $|f|$. Moreover, Rodin-Semenov [17] and Lindenstrauss-Tzafriri [12, pp. 134-138] proved that $\left[r_{n}\right] \simeq l_{2}$ is complemented in $X$ if and only if $G \subset$ $X \subset G^{\prime}$, where $G^{\prime}$ is the Köthe dual space to $G$.

By contrast, Astashkin [2] considered rearrangement invariant spaces $X$ which are interpolation spaces between $G$ and $L_{\infty}$. It turns out that there exists a one-to-one correspondence between them and the sequence spaces $F$ which are interpolation spaces for the couple $\left(l_{1}, l_{2}\right)$; namely, $\left\|\sum_{k=1}^{\infty} a_{k} r_{k}\right\|_{X}$ $\approx\left\|\left\{a_{k}\right\}_{k=1}^{\infty}\right\|_{F}$, where $X=\left(L_{\infty}, G\right)_{\Phi}^{K}$ and $F=\left(l_{1}, l_{2}\right)_{\Phi}^{K}$, with the same parameter space $\Phi$.

Investigations of Rademacher sums in rearrangement invariant spaces are well presented in the books by Lindenstrauss-Tzafriri [12], Kreŭn-PetuninSemenov [10] and Astashkin [3].

The main purpose of this paper is to investigate the behaviour of Rademacher functions in the Cesàro function spaces $\operatorname{Ces}_{p}=\operatorname{Ces}_{p}[0,1]$, which are not rearrangement invariant (cf. 4] and [5], where also other properties are investigated). These spaces are the classes of all Lebesgue measurable real-valued functions $f$ on $[0,1]$ such that

$$
\|f\|_{\operatorname{Ces}_{p}}=\left[\int_{0}^{1}\left(\frac{1}{x} \int_{0}^{x}|f(t)| d t\right)^{p} d x\right]^{1 / p}<\infty \quad \text { for } 1 \leq p<\infty,
$$

and

$$
\|f\|_{\operatorname{Ces} \infty}=\sup _{0<x \leq 1} \frac{1}{x} \int_{0}^{x}|f(t)| d t<\infty \quad \text { for } p=\infty .
$$

We will see that there is an essential difference between the cases $p<\infty$ and $p=\infty$. Namely, for any $1 \leq p<\infty$, the Rademacher functions $\left\{r_{n}\right\}$ span an isomorphic copy of $l_{2}$ in $\operatorname{Ces}_{p}[0,1]$. At the same time, in $\operatorname{Ces}_{\infty}[0,1]$ this system does not contain an unconditional basic subsequence.

In fact, we will consider the Rademacher functions not only in the space $\operatorname{Ces}_{\infty}=: K$, known as the Korenblyum-Krĕn-Levin space [9], but also in more general $K_{p, w}$ spaces or weighted Korenblyum-Krein-Levin spaces.

Let $1 \leq p<\infty$ and $w$ be a quasi-concave function on $I=[0,1]$, that is, $w(0)=0, w$ is non-decreasing, and $w(x) / x$ is non-increasing on $(0,1]$. The spaces $K_{p, w}=K_{p, w}[0,1]$ are the classes of all Lebesgue measurable 
real-valued functions $f$ on $[0,1]$ such that the norm

$$
\|f\|_{K_{p, w}}=\sup _{x \in I, x>0}\left(\frac{1}{w(x)} \int_{0}^{x}|f(t)|^{p} d t\right)^{1 / p}
$$

is finite. These spaces are Banach ideal spaces on $I$. The ideal property means that if $|f| \leq|g|$ a.e. on $I$ and $g \in K_{p, w}$, then $f \in K_{p, w}$ and $\|f\|_{K_{p, w}} \leq$ $\|g\|_{K_{p, w}}$. In the special cases when $w(x)=1$ and $w(x)=x$ we obtain the well-known spaces $L_{p}$ and the Korenblyum-Kreün-Levin spaces $K_{p}$ (see [9]), respectively. Moreover, $K_{1}=K=\operatorname{Ces}_{\infty}$.

For two Banach spaces $X$ and $Y$ the symbol $X \stackrel{C}{\hookrightarrow} Y$ means that the imbedding $X \subset Y$ is continuous with norm not greater than $C$, i.e., $\|x\|_{Y} \leq$ $C\|x\|_{X}$ for all $x \in X$.

2. Rademacher sums in Cesàro type spaces. First we consider the Rademacher sums in the spaces $\operatorname{Ces}_{p}[0,1]$ with $1 \leq p<\infty$.

TheOREM 1. For any $1 \leq p<\infty$, the Rademacher functions $\left\{r_{n}\right\}$ span an isomorphic copy of $l_{2}$ in $\operatorname{Ces}_{p}[0,1]$.

Proof. We need to prove that there are positive constants $A_{p}^{\prime}, B_{p}^{\prime}$ such that

$$
A_{p}^{\prime}\left(\sum_{k=1}^{n} a_{k}^{2}\right)^{1 / 2} \leq\left\|\sum_{k=1}^{n} a_{k} r_{k}\right\|_{\operatorname{Ces}_{p}} \leq B_{p}^{\prime}\left(\sum_{k=1}^{n} a_{k}^{2}\right)^{1 / 2}
$$

for every $n \in \mathbb{N}$ and any real numbers $a_{1}, \ldots, a_{n}$.

Firstly, let us show that the following continuous embeddings hold:

$$
L_{p} \stackrel{p^{\prime}}{\hookrightarrow} \operatorname{Ces}_{p} \stackrel{1}{\hookrightarrow} \operatorname{Ces}_{1}=L_{1}(\ln 1 / t) \stackrel{54}{\hookrightarrow} L_{1 / 3} \quad \text { if } 1<p<\infty,
$$

and

$$
L_{2} \stackrel{\sqrt{2}}{\hookrightarrow} \operatorname{Ces}_{1}=L_{1}(\ln 1 / t) \stackrel{54}{\hookrightarrow} L_{1 / 3} \quad \text { if } p=1 .
$$

The first embedding in (4) is a consequence of the well-known Hardy inequality [7] and the second one follows directly from the fact that $L_{p}[0,1] \stackrel{1}{\hookrightarrow}$ $L_{1}[0,1]$. Since

$$
\int_{0}^{1}\left(\frac{1}{x} \int_{0}^{x}|f(t)| d t\right) d x=\int_{0}^{1}\left(\int_{t}^{1} \frac{1}{x} d x\right)|f(t)| d t=\int_{0}^{1}|f(t)| \ln \frac{1}{t} d t,
$$

we have $\operatorname{Ces}_{1}=L_{1}(\ln 1 / t)$. Assume now $f \in L_{1}(\ln 1 / t)$ with $\|f\|_{L_{1}(\ln 1 / t)}=1$. 
Since $\ln 1 / t \geq 1-t$ if $0<t \leq 1$, it follows that

$$
\begin{aligned}
1=\int_{0}^{1}|f(t)| \ln 1 / t d t & \geq \int_{0}^{1}|f(t)|(1-t) d t \geq \int_{0}^{1} f^{*}(1-t)(1-t) d t \\
& =\int_{0}^{1} f^{*}(s) s d s \geq \int_{0}^{t} f^{*}(s) s d s \geq f^{*}(t) t^{2} / 2,
\end{aligned}
$$

or $f^{*}(t) \leq 2 / t^{2}$. Thus,

$$
\int_{0}^{1}|f(t)|^{1 / 3} d t=\int_{0}^{1} f^{*}(t)^{1 / 3} d t \leq \int_{0}^{1}\left(2 t^{-2}\right)^{1 / 3} d t=3 \cdot 2^{1 / 3}
$$

and so $\|f\|_{L_{1 / 3}} \leq 54$, which finishes the proof of the last imbedding in (4) and (5).

For $p=1$ the first embedding in (5) is a consequence of the HölderRogers inequality

$$
\int_{0}^{1}|f(t)| \ln \frac{1}{t} d t \leq\|f\|_{L_{2}}\left(\int_{0}^{1} \ln ^{2} \frac{1}{t} d t\right)^{1 / 2}=\sqrt{2}\|f\|_{L_{2}},
$$

and the second one is obviously still true for $p=1$.

In view of the Khintchine inequality (1) we obtain $\left\|R_{n}\right\|_{\mathrm{Ces}_{p}} \approx\left\|\left\{a_{k}\right\}_{k=1}^{n}\right\|_{l_{2}}$ or, more precisely,

$$
\frac{A_{1 / 3}}{54}\left\|\left\{a_{k}\right\}_{k=1}^{n}\right\|_{l_{2}} \leq \frac{1}{54}\left\|R_{n}\right\|_{\operatorname{Ces}_{p}} \leq D_{p}\left\|R_{n}\right\|_{L_{p}} \leq D_{p} B_{p}\left\|\left\{a_{k}\right\}_{k=1}^{n}\right\|_{l_{2}},
$$

where $D_{p}=p^{\prime}:=p /(p-1)$ if $p>1$ and $D_{p}=\sqrt{2}$ if $p=1$, and inequalities (3) are proved.

The next result concerns the behaviour of the Rademacher sums in the space $\operatorname{Ces}_{\infty}:=K$ and in its weighted version, i.e., the $K_{p, w}$ space. Our main result is the following:

Theorem 2. For every $1 \leq p<\infty$,

$$
\left\|R_{n}\right\|_{K_{p, w}} \approx\left\|\left\{a_{k}\right\}_{k=1}^{n}\right\|_{l_{2}}+\max _{1 \leq m \leq n}\left(\frac{2^{-m}}{w\left(2^{-m}\right)}\right)^{1 / p}\left|\sum_{k=1}^{m} a_{k}\right| .
$$

Proof. Firstly, we note that it is easy to see that for every $p \geq 1$ and all $m=0,1, \ldots, n$ we have

$$
\int_{0}^{2^{-m}}\left|R_{n}(t)\right|^{p} d t=2^{-n} \sum_{\varepsilon_{k}= \pm 1}\left|a_{1}+\cdots+a_{m}+\varepsilon_{m+1} a_{m+1}+\cdots+\varepsilon_{n} a_{n}\right|^{p},
$$

where the sum is taken over all choices of signs $\varepsilon_{m+1}= \pm 1, \ldots, \varepsilon_{n}= \pm 1$. Therefore, by (2) and by the Hölder-Rogers inequality, for every $m=1, \ldots, n$, 
we obtain

$$
\begin{aligned}
\left\|R_{n}\right\|_{K_{p, w}} & \geq\left(\frac{1}{w\left(2^{-m}\right)} \int_{0}^{2^{-m}}\left|R_{n}(t)\right|^{p} d t\right)^{1 / p} \\
& \geq \frac{2^{m(1-1 / p)}}{w\left(2^{-m}\right)^{1 / p}} \int_{0}^{2^{-m}}\left|R_{n}(t)\right| d t \\
& =\frac{2^{m(1-1 / p)-n}}{w\left(2^{-m}\right)^{1 / p}} \sum_{\varepsilon_{k}= \pm 1}\left|a_{1}+\cdots+a_{m}+\varepsilon_{m+1} a_{m+1}+\cdots+\varepsilon_{n} a_{n}\right| \\
& \geq \frac{2^{m(1-1 / p)-n}}{w\left(2^{-m}\right)^{1 / p}}\left|\sum_{\varepsilon_{k}= \pm 1}\left(a_{1}+\cdots+a_{m}+\varepsilon_{m+1} a_{m+1}+\cdots+\varepsilon_{n} a_{n}\right)\right| \\
& =\frac{2^{m(1-1 / p)-n}}{w\left(2^{-m}\right)^{1 / p}} 2^{n-m}\left|\sum_{k=1}^{m} a_{k}\right|=\left(\frac{2^{-m}}{w\left(2^{-m}\right)}\right)^{1 / p}\left|\sum_{k=1}^{m} a_{k}\right| .
\end{aligned}
$$

Hence, for every $m=1, \ldots, n$,

$$
\left\|R_{n}\right\|_{K_{p, w}} \geq\left(\frac{2^{-m}}{w\left(2^{-m}\right)}\right)^{1 / p}\left|\sum_{k=1}^{m} a_{k}\right| .
$$

On the other hand, by (2) and the classical Khintchine inequality (1), we obtain

$$
\begin{aligned}
\left\|R_{n}\right\|_{K_{p, w}} & \geq \frac{1}{w(1)^{1 / p}} \sup _{0<x \leq 1}\left(\int_{0}^{x}\left|R_{n}(t)\right|^{p} d t\right)^{1 / p} \\
& =\frac{1}{w(1)^{1 / p}}\left\|R_{n}\right\|_{L_{p}[0,1]} \geq \frac{A_{p}}{w(1)^{1 / p}}\left\|\left\{a_{k}\right\}_{k=1}^{n}\right\|_{l_{2}} .
\end{aligned}
$$

This together with (8) implies that

$$
\left\|R_{n}\right\|_{K_{p, w}} \geq C_{p, w}\left(\left\|\left\{a_{k}\right\}_{k=1}^{n}\right\|_{l_{2}}+\max _{1 \leq m \leq n}\left(\frac{2^{-m}}{w\left(2^{-m}\right)}\right)^{1 / p}\left|\sum_{k=1}^{n} a_{k}\right|\right),
$$

where $C_{p, w}=\frac{1}{2} \max \left\{A_{p} w(1)^{-1 / p}, 1\right\}$.

Let us prove the converse inequality. Since $R_{n}(t)$ is a constant on $\left[0,2^{-n}\right]$, we have

$$
\frac{1}{x} \int_{0}^{x}\left|R_{n}(t)\right|^{p} d t=2^{n} \int_{0}^{2^{-n}}\left|R_{n}(t)\right|^{p} d t \quad\left(0<x \leq 2^{-n}\right) .
$$

Moreover, by the quasi-concavity of $w$, one has $x / w(x) \leq 2^{-n} / w\left(2^{-n}\right)$ if $0<x \leq 2^{-n}$ and $w\left(2^{-j}\right) \leq 2 w(x)$ if $x \geq 2^{-j-1}(j=1,2, \ldots)$. Therefore, we 
obtain

$$
\begin{aligned}
& \sup _{x \in(0,1]} \frac{1}{w(x)} \int_{0}^{x}\left|R_{n}(t)\right|^{p} d t \\
& =\max \left[\sup _{x \in\left(0,2^{-n}\right]} \frac{1}{w(x)} \int_{0}^{x}\left|R_{n}(t)\right|^{p} d t, \max _{1 \leq k \leq n} \sup _{x \in\left[2^{-n+k-1}, 2^{-n+k}\right]} \frac{1}{w(x)} \int_{0}^{x}\left|R_{n}(t)\right|^{p} d t\right] \\
& \leq \max \left[\sup _{x \in\left(0,2^{-n}\right]} \frac{x}{w(x)} \frac{1}{x} \int_{0}^{x}\left|R_{n}(t)\right|^{p} d t, \max _{1 \leq k \leq n} \frac{1}{w\left(2^{-n+k-1}\right)} \int_{0}^{2^{-n+k}}\left|R_{n}(t)\right|^{p} d t\right] \\
& \leq \max \left[\frac{1}{w\left(2^{-n}\right)} \int_{0}^{2^{-n}}\left|R_{n}(t)\right|^{p} d t, \max _{1 \leq k \leq n} \frac{2}{w\left(2^{-n+k}\right)} \int_{0}^{2^{-n+k}}\left|R_{n}(t)\right|^{p} d t\right] \\
& \leq 2 \max \left[\frac{1}{w\left(2^{-n}\right)} \int_{0}^{-n}\left|R_{n}(t)\right|^{p} d t, \max _{1 \leq k \leq n} \frac{1}{w\left(2^{-n+k}\right)} \int_{0}\left|R_{n}(t)\right|^{p} d t\right] \\
& =2 \max _{0 \leq k \leq n} \frac{1}{w\left(2^{-n+k}\right)} \int_{0}^{-n+k}\left|R_{n}(t)\right|^{p} d t,
\end{aligned}
$$

and so

$$
\left\|R_{n}\right\|_{K_{p, w}} \leq 2^{1 / p} \max _{0 \leq k \leq n}\left(\frac{1}{w\left(2^{k-n}\right)} \int_{0}^{2^{-n+k}}\left|R_{n}(t)\right|^{p} d t\right)^{1 / p} .
$$

Now, taking into account (7) and using the Minkowski inequality together with the upper estimate from the Khintchine inequality (1), we see that for all $k=0,1, \ldots, n$,

$$
\begin{aligned}
& \left(\int_{0}^{2^{k-n}}\left|R_{n}(t)\right|^{p} d t\right)^{1 / p} \\
& =\left(2^{-n} \sum_{\varepsilon_{i}= \pm 1}\left|a_{1}+\cdots+a_{n-k}+\varepsilon_{n-k+1} a_{n-k+1}+\cdots+\varepsilon_{n} a_{n}\right|^{p}\right)^{1 / p} \\
& \leq\left(2^{-n} \sum_{\varepsilon_{i}= \pm 1}\left|a_{1}+\cdots+a_{n-k}\right|^{p}\right)^{1 / p} \\
& +\left(2^{-n} \sum_{\varepsilon_{i}= \pm 1}\left|\varepsilon_{n-k+1} a_{n-k+1}+\cdots+\varepsilon_{n} a_{n}\right|^{p}\right)^{1 / p} \\
& =2^{(k-n) / p}\left(\left|\sum_{i=1}^{n-k} a_{i}\right|+\left\|\sum_{j=1}^{k} a_{n-k+j} r_{j}\right\|_{L_{p}[0,1]}\right) \\
& \leq 2^{(k-n) / p}\left(\left|\sum_{i=1}^{n-k} a_{i}\right|+B_{p}\left\|\left\{a_{n-k+j}\right\}_{j=1}^{k}\right\|_{l_{2}}\right) \text {. }
\end{aligned}
$$


Therefore, using (9), we get

$$
\begin{aligned}
& \left\|R_{n}\right\|_{K_{p, w}} \leq 2^{1 / p}\left(B_{p} w(1)^{-1 / p}\left\|\left\{a_{k}\right\}_{k=1}^{n}\right\|_{l_{2}}+\max _{1 \leq m \leq n}\left(\frac{2^{-m}}{w\left(2^{-m}\right)}\right)^{1 / p}\left|\sum_{k=1}^{m} a_{k}\right|\right) \\
& \leq 2^{1 / p} \max \left\{B_{p} w(1)^{-1 / p}, 1\right\}\left(\left\|\left\{a_{k}\right\}_{k=1}^{n}\right\|_{l_{2}}+\max _{1 \leq m \leq n}\left(\frac{2^{-m}}{w\left(2^{-m}\right)}\right)^{1 / p}\left|\sum_{k=1}^{m} a_{k}\right|\right),
\end{aligned}
$$

and the proof is complete.

THEOREM 3. The norms $\left\|R_{n}\right\|_{K_{p, w}}$ are uniformly equivalent to the norms $\left\|\left\{a_{k}\right\}_{k=1}^{n}\right\|_{l_{2}}$ with respect to $n \in \mathbb{N}$ if and only if there is a constant $c>0$ such that

$$
w(t) \geq c t \log _{2}^{p / 2}(2 / t) \quad \text { for all } 0<t \leq 1 .
$$

Proof. If 10 holds, then for all $1 \leq m \leq n$ we have

$$
\left(\frac{2^{-m}}{w\left(2^{-m}\right)}\right)^{1 / p} m^{1 / 2} \leq c^{-1 / p}
$$

Since, by the Cauchy-Schwarz inequality,

$$
\left|\sum_{k=1}^{m} a_{k}\right| \leq m^{1 / 2}\left\|\left\{a_{k}\right\}_{k=1}^{m}\right\|_{l_{2}},
$$

(6) and (10) give the required equivalence.

Conversely, suppose that condition (10) does not hold. Then, by the quasi-concavity of $w$, inequality (11) is not satisfied and hence there exists a sequence of natural numbers $m_{k} \rightarrow \infty$ such that

$$
\left(\frac{2^{-m_{k}}}{w\left(2^{-m_{k}}\right)}\right)^{1 / p} m_{k}^{1 / 2} \rightarrow \infty \quad \text { as } k \rightarrow \infty .
$$

Consider the Rademacher sums $R_{k}(t)$ corresponding to the sequences of coefficients $a^{k}=\left(a_{i}^{k}\right)_{i=1}^{m_{k}}$, where $a_{i}^{k}=m_{k}^{-1 / 2}, 1 \leq i \leq m_{k}$. We have $\left\|a^{k}\right\|_{l_{2}}=1$ for all $k=1,2, \ldots$, but $\sum_{i=1}^{m} a_{i}^{k}=m_{k}^{1 / 2}(k=1,2, \ldots)$, which together with (12) and (6) implies that $\left\|R_{k}\right\|_{K_{p, w}} \rightarrow \infty$ as $k \rightarrow \infty$.

REMARK 1 . Let $1 \leq p<\infty$ and let $w$ be a quasi-concave function on $[0,1]$. Consider the $p$-convexification $M_{p, w}$ of the Marcinkiewicz space $M_{1, w}=M(w)$ with the norm given by

$$
\|f\|_{M_{p, w}}=\sup _{0<x \leq 1}\left(\frac{1}{w(x)} \int_{0}^{x} f^{*}(t)^{p} d t\right)^{1 / p} .
$$

The space $M_{p, w}$ can be treated as a rearrangement invariant version of $K_{p, w}$. It is not hard to check that the embedding $G \subset M_{p, w}$ (see Section 1) holds if and only if the condition (10) is satisfied. Therefore, by Theorem 3 and by 
the result of Rodin-Semenov [16] (see also [12, Th. 2.b.4]), it follows that the Rademacher functions span $l_{2}$ in $M_{p, w}$ if and only if they span $l_{2}$ in $K_{p, w}$.

Moreover, it is instructive to compare the behaviour of Rademacher sums in the spaces $M_{1, w}=M(w)$ and $K_{1, w}$ in the case when $w(t)=t \log _{2}^{1 / q}(2 / t)$, where $q>2$. Then (10) does not hold and, by (6),

$$
\left\|R_{n}\right\|_{K_{1, w}} \approx\left\|\left\{a_{k}\right\}_{k=1}^{n}\right\|_{l_{2}}+\max _{1 \leq m \leq n} m^{-1 / q}\left|\sum_{k=1}^{m} a_{k}\right| .
$$

On the other hand, by the result of Rodin-Semenov [16, p. 221] and Pisier [15] (see also Marcus-Pisier [14, pp. 277-278]), the norm $\left\|R_{n}\right\|_{M(w)}$ is equivalent to the norm of the sequence of coefficients in the Marcinkiewicz sequence space $l_{q, \infty}$ given by

$$
\left\|\left\{a_{k}\right\}\right\|_{l_{q, \infty}}=\sup _{m=1,2, \ldots} m^{-1 / q} \sum_{k=1}^{m} a_{k}^{*},
$$

where $\left\{a_{k}^{*}\right\}$ is the non-increasing rearrangement of $\left\{\left|a_{k}\right|\right\}_{k=1}^{\infty}$.

The following corollaries follow directly from Theorem 1 in the special case $w(x)=x$.

COROllary 1. For any $1 \leq p<\infty$ we have the equivalence

$$
\left\|R_{n}\right\|_{K_{p}} \approx\left\|\left\{a_{k}\right\}_{k=1}^{n}\right\|_{l_{2}}+\max _{1 \leq m \leq n}\left|\sum_{k=1}^{m} a_{k}\right|,
$$

and, in particular, the same holds for the space $\operatorname{Ces}_{\infty}[0,1]=K_{1}$.

COROllary 2. Let $1 \leq p<\infty$. The series $\sum_{k=1}^{\infty} a_{k} r_{k}$ is convergent in $K_{p}$ if and only if $\sum_{k=1}^{\infty} a_{k}^{2}<\infty$ and the series $\sum_{k=1}^{\infty} a_{k}$ is convergent.

COROLlary 3. The sequence of Rademacher functions is equivalent in $K_{p}$ to each of its subsequences.

COROLlaRY 4. The system of Rademacher functions does not contain an unconditional basic subsequence in $K_{p}$.

Remark 2. From (13) we obtain

$$
\left\|\sum_{k=1}^{\infty} a_{k} r_{k}\right\|_{K_{p}} \approx\left\|\left\{a_{k}\right\}_{k=1}^{\infty}\right\|_{l_{2}}+\sup _{m \in \mathbb{N}}\left|\sum_{k=1}^{m} a_{k}\right| .
$$

A similar equivalence holds also for the BMO space on [0, 1]. In 1985 Mikhail Lelbov showed in his $\mathrm{PhD}$ dissertation [11] that

$$
\left\|\sum_{k=1}^{\infty} a_{k} r_{k}\right\|_{\mathrm{BMO}} \approx\left\|\left\{a_{k}\right\}_{k=1}^{\infty}\right\|_{l_{2}}+\sup _{l, m \in \mathbb{N}, l \leq m}\left|\sum_{k=l}^{m} a_{k}\right| .
$$


Noting that

$$
\begin{aligned}
\sup _{m \in \mathbb{N}}\left|\sum_{k=1}^{m} a_{k}\right| & \leq \sup _{l, m \in \mathbb{N}, l \leq m}\left|\sum_{k=l}^{m} a_{k}\right| \\
& =\sup _{l, m \in \mathbb{N}, l \leq m}\left|\sum_{k=1}^{m} a_{k}-\sum_{k=1}^{l-1} a_{k}\right| \leq 2 \sup _{m \in \mathbb{N}}\left|\sum_{k=1}^{m} a_{k}\right|,
\end{aligned}
$$

we obtain estimates which are similar to (14) but with BMO instead of $K_{p}$. Note that the spaces $K_{p}$ and BMO are not comparable, that is, no one of them is embedded in the other.

\section{Complementability of Rademacher subspaces in Cesàro type} spaces. We first consider the problem of complementability of the closed linear span $\left[r_{n}\right]_{n=1}^{\infty}$ in the spaces $K_{p, w}$. We begin with the case when $p=1$.

THEOREM 4. For every quasi-concave function $w$, the subspace $\left[r_{n}\right]_{n=1}^{\infty}$ of the Banach space $K_{1, w}$ is not complemented in that space.

Proof. We will consider only the case when $\lim _{x \rightarrow 0+} x / w(x)>0$ (in other words, when $K_{1, w}$ coincides with the Korenblyum-Kreln-Levin space $K:=$ $\operatorname{Ces}_{\infty}[0,1]$ with equivalence of norms) because the proof in the remaining case when $\lim _{x \rightarrow 0+} x / w(x)=0$ is completely similar and even a little easier.

Suppose that $\left[r_{n}\right]_{n=1}^{\infty}$ is a complemented subspace of $K$, and $P$ is the corresponding bounded linear projector. Since, by Theorem 2 , the Rademacher functions form a basic sequence in $K$, there exist functionals $\phi_{n} \in K^{*}$ $(n=1,2, \ldots)$ such that

$$
P f(x)=\sum_{n=1}^{\infty} \phi_{n}(f) r_{n}(x) \quad(f \in K) .
$$

By [13, the Köthe dual $K^{\prime}$ equals $\tilde{L_{1}}$ with equality of norms, where

$$
\|f\|_{\tilde{L_{1}}}=\|\tilde{f}\|_{L_{1}}, \quad \text { with } \quad \tilde{f}(x)=\operatorname{ess~sup}_{t \in[x, 1]}|f(t)| .
$$

Since $\tilde{L_{1}}$ is a total set in $K$, by [8, Ch. 10, Theorem 3.6, Russian edition],

$$
K^{*}=K^{\prime} \oplus\left(K^{\prime}\right)^{d} \text {, }
$$

where $\left(K^{\prime}\right)^{d}$ is the set of all singular bounded linear functionals on $K$. In particular, if $\theta \in\left(K^{\prime}\right)^{d}$, then

$$
\theta(f)=0 \quad \text { for every } f \in K_{0},
$$

where $K_{0}$ is the separable part of $K$.

Equality (16) implies that $\phi_{n}=\psi_{n}+\theta_{n}$, where $\psi_{n} \in K^{\prime}$ and $\theta_{n} \in\left(K^{\prime}\right)^{d}$ $(n=1,2, \ldots)$. Moreover, since $P$ is a projection onto $\left[r_{n}\right]_{n=1}^{\infty}$,

$$
\psi_{n}\left(r_{n}\right)+\theta_{n}\left(r_{n}\right)=1 \text { and } \psi_{n}\left(r_{i}\right)+\theta_{n}\left(r_{i}\right)=0 \quad \text { if } i \neq n .
$$


By (17), $\theta_{n}\left(r_{i}\right)=\theta_{n}\left(\chi_{[0,1]}\right):=c_{n}$ for all positive integers $n$ and $i$. Therefore, (18) implies that $\psi_{n}\left(r_{i}\right)=-c_{n}$ for all $i \neq n$. On the other hand,

$$
\psi_{n}(f)=\int_{0}^{1} g_{n}(t) f(t) d t,
$$

where $g_{n} \in \tilde{L_{1}}(n=1,2, \ldots)$. Taking into account that $\tilde{L_{1}} \subset L_{1}$ and that $\left\{r_{i}\right\}$ is a uniformly bounded orthonormal sequence of functions we have $\psi_{n}\left(r_{i}\right) \rightarrow 0$ as $i \rightarrow \infty$ for every $n=1,2, \ldots$ Therefore, $c_{n}=0(n=1,2, \ldots)$, and, by $(18)$ and $(19)$, we conclude that

$$
\int_{0}^{1} g_{n}(t) r_{n}(t) d t=1 \quad \text { and } \quad \int_{0}^{1} g_{n}(t) r_{i}(t) d t=0 \quad \text { if } i \neq n .
$$

Moreover, the restriction of the projection $P$ from 15 to the separable part $K_{0}$ (we will denote it in the same way) may be represented in the form

$$
P f(x)=\sum_{n=1}^{\infty} \int_{0}^{1} g_{n}(t) f(t) d t r_{n}(x) \quad\left(f \in K_{0}\right) .
$$

Next, we note that there exist a small enough $h \in(0,1)$ and a positive integer $n_{0}$ such that for all $n \geq n_{0}$,

$$
\left|\int_{h}^{1} g_{n}(t) r_{n}(t) d t\right| \geq \frac{1}{2}
$$

In fact, otherwise, 20 implies that there is a subsequence $\left\{g_{n_{k}}\right\} \subset\left\{g_{n}\right\}$ satisfying

$$
\int_{0}^{1 / i}\left|g_{n_{i}}(t)\right| d t \geq\left|\int_{0}^{1 / i} g_{n_{i}}(t) r_{n_{i}}(t) d t\right| \geq \frac{1}{2} \quad(i=1,2, \ldots) .
$$

By Theorem 1, for every $f \in L_{\infty} \subset K$ we have

$$
\sum_{n=1}^{\infty}\left(\int_{0}^{1} g_{n}(t) f(t) d t\right)^{2}<\infty
$$

which implies that $g_{n} \stackrel{w}{\longrightarrow} 0$ in $L_{1}[0,1]$. Therefore, by the Dunford-Pettis criterion [1, Theorem 5.2.9], $\left\{g_{n}\right\}_{n=1}^{\infty}$ is an equi-integrable set in $L_{1}[0,1]$, which contradicts (22). Thus, inequalities (21) hold for all $n \geq n_{0}$.

Now consider the operator $P_{h}$ defined by

$$
P_{h} f(x)=\sum_{n=1}^{\infty} \int_{h}^{1} g_{n}(t) f(t) d t r_{n}(x) .
$$


Since, by Theorem 2 and the classical Khintchine inequality (1),

$$
\left\|\sum_{n=1}^{\infty} a_{n} r_{n}\right\|_{L_{1}[0,1]} \leq C\left\|\sum_{n=1}^{\infty} a_{n} r_{n}\right\|_{K},
$$

$P_{h}$ acts boundedly from $K$ into $L_{1}[0,1]$. At the same time, if $f$ is a measurable function on $[0,1]$ such that $\operatorname{supp} f \subset[h, 1]$, then

$$
\|f\|_{L_{1}[h, 1]} \leq\|f\|_{K}=\sup _{h \leq x \leq 1} \frac{1}{x} \int_{x}^{1}|f(t)| d t \leq \frac{1}{h}\|f\|_{L_{1}[h, 1]},
$$

and therefore $P_{h}: L_{1}[h, 1] \rightarrow L_{1}[0,1]$ is bounded. Moreover, by the definition of the operator $P_{h}$ and inequality (1), we see that $P_{h}$ is weakly compact. Since the space $L_{1}[h, 1]$ has the Dunford-Pettis property [1, Theorem 5.4.5] it follows that $P_{h}$ is weak-to-norm sequentially continuous. Therefore, taking into account that $r_{n} \chi_{[h, 1]} \stackrel{w}{\rightarrow} 0$ in $L_{1}[h, 1]$, we find that $\left\|P_{h}\left(r_{n} \chi_{[h, 1]}\right)\right\|_{L_{1}[0,1]}$ $\rightarrow 0$. On the other hand, by (1) and (21),

$$
\left\|P_{h}\left(r_{n} \chi_{[h, 1]}\right)\right\|_{L_{1}[0,1]} \asymp\left(\sum_{i=1}^{\infty}\left(\int_{h}^{1} g_{i}(t) r_{n}(t) d t\right)^{2}\right)^{1 / 2} \geq\left|\int_{h}^{1} g_{n}(t) r_{n}(t) d t\right| \geq \frac{1}{2},
$$

for all $n \geq n_{0}$. This contradiction concludes the proof.

In the case $p>1$ the situation is completely different.

TheOREM 5. Let $1<p<\infty$. If the weight $w$ satisfies condition 10, then the subspace $\left[r_{n}\right]_{n=1}^{\infty}$ is complemented in $K_{p, w}$.

Proof. By [17] (cf. [12, pp. 134-138]), the orthogonal projection

$$
Q f(x)=\sum_{n=1}^{\infty} \int_{0}^{1} r_{n}(t) f(t) d t r_{n}(x)
$$

is bounded in $L_{p}[0,1](1<p<\infty)$. Therefore, Theorem 3 yields

$$
\|Q f\|_{K_{p, w}} \leq C\|Q f\|_{L_{p}} \leq C\|Q\|_{L_{p} \rightarrow L_{p}}\|f\|_{L_{p}} \leq C\|Q\|_{L_{p} \rightarrow L_{p}} w(1)^{1 / p}\|f\|_{K_{p, w}} \text {, }
$$

which implies that $Q$ is bounded in $K_{p, w}$.

REMARK 3 . In contrast to the spaces $K_{1, w}$, the subspace $\left[r_{n}\right]_{n=1}^{\infty}$ is complemented in a Marcinkiewicz space $M_{1, w}=M(w)$ if and only if $G \subset$ $M(w) \subset G^{\prime}$ (cf. [17] and [12, pp. 134-138]). Note that the left hand embedding is equivalent to condition (10).

Finally, let us consider the case of Cesàro spaces with finite $p$. First we note that, in contrast to $K=\operatorname{Ces}_{\infty}[0,1]$, the spaces $\operatorname{Ces}_{p}[0,1](1 \leq p<\infty)$ are separable. This implies that $\operatorname{Ces}_{p}[0,1]^{*}$ coincides with the Köthe dual $\operatorname{Ces}_{p}[0,1]^{\prime}$ (described in [4]). It is also easy to see that for any $h \in(0,1)$ there is a constant $C_{h}>0$ such that for every measurable function $f$ on $[0,1]$ with supp $f \subset[h, 1]$, we have $\|f\|_{\operatorname{Ces}_{p}} \leq C_{h}\|f\|_{L_{1}}$. Therefore, by Theorem 1, 
arguing in the same way as in the proof of Theorem 4, we can prove the following result:

TheOREM 6. For any $1 \leq p<\infty$, the subspace $\left[r_{n}\right]$ is not complemented in $\operatorname{Ces}_{p}[0,1]$.

Acknowledgments. Research of S. V. Astashkin was partially supported by the Ministry of Education and Science of the Russian Federation grant 3341.

Research of L. Maligranda was partially supported by the Swedish Research Council (VR) grant 621-2008-5058.

\section{References}

[1] F. Albiac and N. J. Kalton, Topics in Banach Space Theory, Grad. Texts in Math. 233, Springer, New York, 2006.

[2] S. V. Astashkin, About interpolation of subspaces of rearrangement invariant spaces generated by Rademacher system, Int. J. Math. Math. Sci. 25 (2001), 451-465.

[3] - Rademacher functions in symmetric spaces, Contemporary Mathematics. Fundamental Directions, Russian Peoples' Friendship University 32 (2009), 3-161 (in Russian).

[4] S. V. Astashkin and L. Maligranda, Geometry of Cesàro function spaces, Funktsional. Anal. i Prilozhen., to appear.

[5] —, -, Structure of Cesàro function spaces, Indag. Math. (N.S.) 20 (2009), 329-379.

[6] J. Diestel, H. Jarchow and A. Tonge, Absolutely Summing Operators, Cambridge Univ. Press, Cambridge 1995.

[7] G. H. Hardy, J. E. Littlewood and G. Pólya, Inequalities, Cambridge Univ. Press, 1952.

[8] L. V. Kantorovich and G. P. Akilov, Functional Analysis, Nauka, Moscow, 1977 (in Russian); English transl.: Pergamon Press, New York, 1982.

[9] B. I. Korenblyum, S. G. KreĬn and B. Ya. Levin, On certain nonlinear questions of the theory of singular integrals, Dokl. Akad. Nauk SSSR 62 (1948), 17-20 (in Russian).

[10] S. G. Krĕ̌n, Yu. I. Petunin, and E. M. Semenov, Interpolation of Linear Operators, Nauka, Moscow, 1978 (in Russian); English transl.: Amer. Math. Soc., Providence, 1982.

[11] M. V. Leibov, The geometry of a functional space, Candidate's Dissertation, Rostov na Donu, 1985 (in Russian).

[12] J. Lindenstrauss and L. Tzafriri, Classical Banach Spaces, II. Function Spaces, Springer, Berlin, 1979.

[13] W. A. J. Luxemburg and A. C. Zaanen, Some examples of normed Köthe spaces, Math. Ann. 162 (1965/1966), 337-350.

[14] M. B. Marcus and G. Pisier, Characterizations of almost surely continuous p-stable random Fourier series and strongly stationary processes, Acta Math. 152 (1984), 245-301.

[15] G. Pisier, De nouvelles caractérisations des ensembles de Sidon, in: Mathematical Analysis and Applications, Part B, Adv. Math. Suppl. Stud. 7b, Academic Press, New York, 1981, 685-726. 
[16] V. A. Rodin and E. M. Semyonov, Rademacher series in symmetric spaces, Anal. Math. 1 (1975), 207-222.

[17] - - - The complementability of a subspace that is generated by the Rademacher system in a symmetric space, Funktsional. Anal. i Prilozhen. 13 (1979), no. 2, 91-92 (in Russian); English transl.: Funct. Anal. Appl. 13 (1979), no. 2, 150-151.

Sergei V. Astashkin

Department of Mathematics and Mechanics

Lech Maligranda

Samara State University

Akad. Pavlova 1

443011 Samara, Russia

E-mail: astashkn@ssu.samara.ru

Department of Mathematics Luleå University of Technology SE-971 87 Luleå, Sweden E-mail: lech@sm.luth.se

Received August 18, 2009

Revised version February 3, 2010 